\title{
Tagungsbericht
}

Anne-Katharina Weilenmann*

\section{Die differenzierten Facetten des Informationsverhaltens}

\author{
Tagungsbericht ISIC-Konferenz 2016
}

DOI 10.1515/iwp-2017-0020

\begin{abstract}
„Man weiss heute, dass nur ein winziger Bruchteil der Informationen, die der Körper über seine Sinne aufnimmt, in unsere bewusste Wahrnehmung gelangt. Von 10 Millionen Bits, die wir aufnehmen, verarbeiten wir eine Million und nutzen schließlich lediglich deren $50 . " 1$
\end{abstract}

Die zunehmende Technisierung der Welt schafft ungeahnte Möglichkeiten, durchdringt unmerklich unseren Alltag. Neuartige, innovative Anwendungen, kleine technische Hilfsmittel nehmen uns (unliebsame) Aufgaben ab, erleichtern es uns, die täglichen Arbeiten zu erledigen. Schlagworte wie Internet der Dinge, künstliche Intelligenz, Arbeit 4.0, digitale Bildung sind fast tagtäglich der Presse zu entnehmen, stehen im Fokus der allgemeinen (politischen) Diskussion. Doch wie verhält sich der Mensch in einem Umfeld, das nicht nur immer komplexer wird, sondern ihm gleichzeitig suggeriert, seine Lebenswelt einfacher zu gestalten?

Mit diesen Fragestellungen befasst sich die „Information Behaviour Conference“ (ISIC). ${ }^{2}$ Neben internationalen und europäischen Konferenzen, ${ }^{3}$ die regelmäßig zum allgegenwärtig präsenten Thema von Informations- und Medienkompetenz stattfinden, ist die ISIC damit eine der wenigen Veranstaltungen, die sich im Zwei-Jahresrhythmus dem Informationsverhalten widmet. Die letzte ISICKonferenz fand vom 20. bis 23. September 2016 in Zadar

1 siehe Seydel, 2001.

2 http://www.isic2016.com [18.12.2016].

3 zu den wichtigsten gehören hier: Librarians' Information Literacy Annual Conference (LILAC): http://www.lilacconference.com [18.12. 2016] sowie die European Conference on Information Literacy (ECIL): http://www.ilconf.org/ [18.12.2016].

*Kontaktperson: Anne-Katharina Weilenmann, Stettemerstrasse 165, 8207 Schaffhausen, Schweiz, E-Mail: weilenmann@shinternet.ch, http://www.biblink.ch, ORCID: 0000-0002-4950-9866 statt und stand unter dem Motto „Information Seeking in Context".4

Dem Konferenzpublikum wurde in einer frühherbstlich angenehm warmen Umgebung ein facettenreiches Programm geboten. Der formale Rahmen der vier Kongresstage gliederte sich in die Vorkonferenz mit den „Doctoral Workshops und Presentations“5 sowie ins Hauptprogramm mit den Vortrags- und Postersessions. ${ }^{6}$ Inhaltlich ließ sich das Programm grob den fünf Hauptbereichen Theorien/Modelle, Informationen generieren/ suchen/teilen, Social Networks, arbeitsbezogene Informationspraktiken sowie gesundheitsbezogene Informationssuche zuordnen; dies war wiederum unterteilt in folgende Sessions: Theories and models of information behaviour, Methodological issues of information behaviour research, Information sharing and seeking in diverse contexts, Knowledge creation and information intermediation, Social media and social networks in information behaviour, Information searching, Work-related information practices, Searching and reading behaviour, Contexts of information sharing, Health information seeking und Information seeking in learning contexts.

4 Das Gesamtprogramm kann unter http://isic2016.com/?page id=176 [18.12.2016] eingesehen werden; die Conference-Proceedings werden in zwei Teilen in einer Sonderausgabe der Zeitschrift „Information Research“" publiziert, http://www.informationr.net [18.12. 2016]; der erste Teil der Proceedings ist online unter: http://www. informationr.net/ir/21-4/isic/isic2016.html [18.12.2016].

5 das ausführliche Programm dazu befindet sich unter http:// isic2016.com/?page_id=989 [18.12.2016].

6 Liste mit den „Accepted Papers“: http://isic2016.com/?page_ id=1009 [18.12.2016], Liste mit den „Accepted Poster Preposals“: http://isic2016.com/?page_id=1033 [18.12.2016]. 


\section{Von Theoriebildung bis zu Informationspraktiken und 3D-Modellierung}

„While some researchers use "information behavior” narrowly to refer only to information seeking activities, a majority follow Wilson's (1999, p. 249) encapsulation that information behavior is "the totality of human behavior in relation to sources and channels of information, including both active and passive information seeking, and information use.” It was along this line that Pettigrew, et al., (2001, p. 44) defined information behavior as "how people need, seek, give and use information in different contexts. ${ }^{\text {"7 }}$ Diese wohlbekannte Definition des Begriffs „Informationsverhalten“ spiegelte die Grundstimmung und Motivation der Konferenzteilnehmerinnen und -teilnehmer wider und zog sich wie ein roter Faden durch die einzelnen Präsentationen, Gespräche und Diskussionen.

Passend dazu beleuchtete Wilson in seinem Vortrag „A general theory of information behaviour"8 die von ihm entwickelten Modelle zum Informationsverhalten. Mit einer Untersuchung $\mathrm{zu} 20$ Jahren ISIC-Forschung befasste sich die Autorengruppe um McKechnie. Analysiert wurden 193 Artikel, die im Zeitraum von 1996 bis 2014 in den ISICProceedings publiziert worden sind. Die Forschergruppe kam zum Schluss, dass nur knapp ein Viertel (22.8 \%) der Artikel genügend Daten zur Replikation der Forschungsergebnisse enthalten. ${ }^{9}$ Neben diesem theoretischen Teil zu Theorie und Methoden, der insgesamt sechs Präsentationen enthielt, waren die weiteren Ausführungen eher praxisorientiert und erlaubten Einblicke in einzelne Forschungsprojekte und in das Informationsverhalten unterschiedlichster Zielgruppen (wie z. B. clean energy markets, \#Homeless oder Pacific Crest Trail thru-hikers). Es fällt auf, dass Präsentationen im Kontext von Gesundheit und Information stark vertreten sind.

\section{Postersession}

In den Postersessions wurden 17 aufwändig gestaltete Posters gezeigt. Man traf auf ein breit gefächertes Themenspektrum; die Vielfalt reichte von E-Health-Dienstleistungen über Online-Journalismus bis zu Immigrantinnen aus

7 siehe Theories of Information behavior, 2009.

8 publiziert im ersten Teil der Proceedings: http://www.informationr.net/ir/21-4/isic/isic1601.html [18.12.2016].

9 Die Studie kann hier nachgelesen werden: http://www.informationr.net/ir/21-4/isic/isic1604.html [18.12.2016].
Bangladesh. Die Postersession zeigte sich als ein lebendiges Forum zum Austausch von Ideen und angeregten Diskussionen.

\section{Informationswissenschaft und Neurowissenschaft - ein Zusammenspiel?}

Vor dem Hintergrund aktueller Entwicklungen in der Bildungstechnologie ${ }^{10}$ und den Mediengewohnheiten der Millennials mag es erstaunen, dass dieser Thematik auf der Konferenz kaum Raum gegeben wurde.

„Reading a book is last century." ${ }^{11}$ Diese Aussage eines Millennials lässt doch aufhorchen. Wie verändert sich unser Lern- und Arbeitsverhalten mit zunehmender Technisierung, wie lernen die Generationen Y und Z? Welche Kompetenzen werden (noch) benötigt? Wie kann die Informationswissenschaft dazu beitragen, solche Fragen zu erforschen, welcher Instrumente und Methoden bedarf es, um das Informationsverhalten der (jungen) Generationen zu untersuchen?

Ein interdisziplinärer Ansatz dazu wurde von der Autorin in der Postersession „Information Science and Neuroscience - an interaction?"“12 präsentiert. Dabei standen folgende zwei Aspekte im Mittelpunkt:

a) Müssen Informationswissenschaftlerinnen und Informationswissenschaftler über grundlegende neurologische Kenntnisse verfügen?

b) Ist es notwendig, neurologisches Grundlagenwissen in die informationswissenschaftliche Ausbildung zu integrieren?

In der Fachliteratur ist der Einsatz neurologischer Methoden kaum dokumentiert. ${ }^{13}$ Seit einiger Zeit ist jedoch eine neue Bewegung erkennbar, die seit 2010 unter dem Namen „Gmunden Retreat“14 jährliche Konferenzen veranstaltet, mit dem Ziel, den Diskurs zwischen Informationswissenschaft und Neurowissenschaft zu fördern. Mit dem Ausdruck „Neuro-Information-Systems“ (NeuroIS) schaffen Riedl und Léger (2016) eine neue Forschungsdisziplin und

10 wie z.B. der Einsatz von Virtuellen Technologien im Klassenzimmer als „Virtual Classroom“: http://www.imsimity.de [18.12.2016]. 11 siehe Cuende 2016.

12 Abstract: http://isic2016.com/wp-content/uploads/2016/10/Wei lenmann-Information-Science-and-Neuroscience-an-interaction.pdf [18.12.2016].

13 siehe dazu Ferebee und Davis, 2011.

14 http://www.neurois.org [18.12.2016]. 
definieren diese wie folgt: ,....an interdisciplinary field of research that relies on knowledge from disciplines related to neurobiology and behavior, as well as knowledge from engineering disciplines. “15 Doch wie könnten nun Methoden und Werkzeuge aus dieser Disziplin in der Informationswissenschaft angewendet werden? Denkbar wäre u.a. der Einsatz von Elektrodermaler Aktivität (EDA) ${ }^{16}$ bei PeerReviewing-Verfahren; die Reaktionen des Peer Reviewers könnten so als erster Eindruck eines Artikels verwendet werden...

Informationswissenschaft und Neurowissenschaft als interdisziplinäres Zusammenspiel? - ein innovativer Schritt in die Zukunft: die Neuro-Informationswissenschaft.

\section{Fazit}

Die ISIC-Konferenz 2016 vermittelte umfangreiche neue Informationen, Impulse und Anregungen, das weite Forschungsfeld von „Information behavior“ zu untersuchen und bot letztlich auch Gelegenheit, das eigene Informationsverhalten kritisch zu beleuchten.

Deskriptoren: Tagung, Informationsverhalten, Informationswissenschaft, Hirnforschung, ISIC

\section{Literatur}

Cuende, L. I., zitiert als: Dugall, B.: The Selfie Generation: 7th OCLC EMEA Regional Council Meeting, 1. und 2. März 2016. In: ABI Technik, Vol. 36(2016), Nr. 2, S. 114-119. DOI 10.1515/abitech2016-0018.

Ferebee, S.; Davis, J. W.: Persuading library use in technologically structured individuals. In: Library Hi Tech, Vol. 29(2011), No. 4, S. 586-604. DOI 10.1108/07378831111189714.

Pettigrew, K.; Fidel, R.; Bruce, H.: Conceptual frameworks in information behavior. Annual Review of Information Science and Technology, 35(2001), S. 43-78.

Riedl, R.; Léger, P.-M.: Fundamentals of NeurolS: Information Systems and the Brain. Berlin, Heidelberg : Springer, 2016.

Seydel, S. M.: Die Welt ist weder Scheibe noch Kugel - sie ist ein Gespräch. In: Der StellenBund, Jg. 152, Nr. 185 vom 11. August 2001.

Theories of information behavior. ed. by K. E. Fisher ... [et al.], 3rd ed.: Medford, N.J. : Information Today, 2009.

Wilson, T. D.: Models in information behaviour research. Journal of Documentation, 55(1999), no 3, S. 249-270.

15 siehe Riedl und Léger, 2016.

16 Messen des Hautwiderstands; wird z. B. in der Stress- und Emotionsforschung eingesetzt

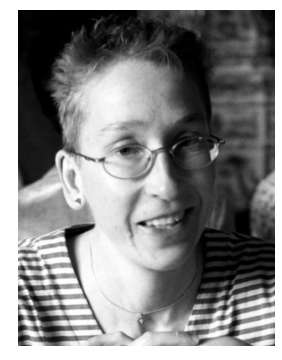

Anne-Katharina Weilenmann Stettemerstrasse 165 8207 Schaffhausen Schweiz weilenmann@shinternet.ch http://www.biblink.ch ORCID: 0000-0002-4950-9866

Anne-Katharina Weilenmann hat einen MSc Bibliotheks- und Informationsmanagement der Donau-Universität Krems. Sie arbeitet freiberuflich als Referentin; ist Dozentin für Internet- und Onlinerecherche an der Haute école de gestion de Genève - HEG und ist zurzeit mit ihrem persönlichen Dissertationsprojekt zur Dekonstruktion des wissenschaftlichen Artikels beschäftigt. 